
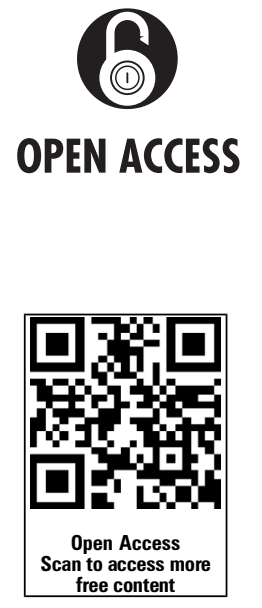

${ }^{1}$ Nottingham Digestive Diseases Centre and NIHR Biomedical Research Unit, Nottingham University Hospitals NHS Trust and University of Nottingham, Nottingham, UK

${ }^{2}$ Digestive Diseases Centre, University Hospitals of Leicester NHS Trust, Leicester, UK

${ }^{3}$ Gastroenterology and Hepatology Department, Derby Hospitals NHS Foundation Trust, Derby, UK

\section{Correspondence to} Professor Krish Ragunath MD FRCP FASGE, Professor \& Head of GI Endoscopy, Nottingham Digestive Diseases Centre, NIHR Biomedical Research Unit, Queens Medical Centre campus, Nottingham University Hospitals NHS Trust, Nottingham NG7 2UH, UK; K.Ragunath@nottingham.ac.uk

Received 7 January 2015 Revised 5 February 2015 Accepted 9 February 2015 Published Online First 13 March 2015

\section{SLinked}

- http://dx.doi.org/10.1136/ flgastro-2015-100584

CrossMark

To cite: Ortiz-FernándezSordo J, Sami S, MansillaVivar $\mathrm{R}$, et al. Frontline Gastroenterology 2016;7: 24-29.

\title{
Incidence of metachronous visible
} lesions in patients referred for radiofrequency ablation (RFA) therapy for early Barrett's neoplasia: a single-centre experience

\author{
J Ortiz-Fernández-Sordo, ${ }^{1}$ S Sami, ${ }^{1}$ R Mansilla-Vivar, ${ }^{1}$ J De Caestecker, ${ }^{2}$ \\ A Cole, ${ }^{3} \mathrm{~K}$ Ragunath ${ }^{1}$
}

\begin{abstract}
Objective Evaluate the incidence of metachronous visible lesions (VLS) in patients referred for radiofrequency ablation (RFA) for early Barrett's neoplasia.
\end{abstract}

Design This study was conducted as part of the service evaluation audit.

Setting Tertiary referral centre.

Patients All patients with dysplastic Barrett's oesophagus referred for RFA were included for analysis. White light high-resolution endoscopy (HRE), autofluorescence imaging and narrow band imaging were sequentially performed. Endoscopic mucosal resection (EMR) was performed for all VL. Three to six months after EMR, all patients underwent initial RFA and then repeat RFA procedures at three monthly intervals. Interventions All endoscopy reports and final staging by EMR/surgery were evaluated and included for analysis.

Results Fifty patients were analysed; median age 73 years, $84 \%$ men. 38/50 patients (76\%) had a previous EMR due to the presence of $\mathrm{VL}$ before referred for ablation; twelve patients had no previous treatment. In total, 151 ablation procedures were performed, median per patient 2.68. Twenty metachronous VL were identified in 14 patients before the first ablation or during the RFA protocol; incidence was $28 \%$. All

metachronous lesions were successfully resected by EMR. Upstaging after rescue EMR compared with the initial histology was observed in four patients (28\%).

Conclusions In total, $28 \%$ of patients enrolled in the RFA programme were diagnosed to have metachronous lesions. This high-incidence rate highlights the importance of a meticulous examination to identify and resect any VL before every ablation session. RFA treatment for early Barrett's neoplasia should be performed in tertiary referral centres with HRE and EMR facilities and expertise.

\section{INTRODUCTION}

Barrett's oesophagus (BO) with highgrade dysplasia (HGD) is the most important risk factor for developing oesophageal adenocarcinoma, the most rapidly increasing cancer in western countries. The global incidence of oesophageal cancer arising from $\mathrm{BO}$ is $0.5 \%$ per year ${ }^{1} 2$ and increases up to $10 \%$ yearly in patients with $\mathrm{BO}$ and HGD. ${ }^{3}$

In $\mathrm{BO}$, the normal squamous mucosa is replaced by columnar epithelium with the presence of intestinal metaplasia (IM). The malignant transformation of BO from non-dysplastic IM to invasive adenocarcinoma is a gradual process through well-known sequential histological stages: low-grade dysplasia (LGD), HGD and early intramucosal cancer (IMC/T1a).

All these early lesions have a negligible risk of lymph node metastases, which is clearly related to the depth of tumour infiltration in the oesophageal wall. ${ }^{4} 5$ The incidence of lymph node metastases is between $0 \%$ and $3 \%$ for lesions limited to the mucosa $(\mathrm{T} 1 \mathrm{a} / \mathrm{m} 1-\mathrm{m} 3)$, but rises up to $30 \%$ when the lesion is invading the submucosal (SM) layer (T1b/sm1$\operatorname{sm} 3){ }^{6} 7$ 
Despite the lack of randomised controlled trials and cost-effectiveness analysis, endoscopic surveillance programmes with targeted biopsies from any visible lesion $(\mathrm{VL})$ and random four-quadratic biopsies have been shown to detect the presence of neoplasia at a potentially curative stage. ${ }^{8}$ Most of the current clinical guidelines developed by gastrointestinal societies recommend regular endoscopic surveillance for Barrett's patients to detect neoplastic lesions in an early stage. ${ }^{9}$

These early neoplastic lesions are difficult to identify with conventional white light endoscopy ${ }^{10}$ and other diagnostic tools, such endoscopic ultrasound, have shown to have virtually no clinical impact on the workup of early oesophageal neoplasia ${ }^{11}$ because its lack of accuracy to differentiate between mucosal and SM invasion in early oesophageal lesions. ${ }^{12} 13$

In addition to the white light high-resolution endoscopy (HRE), the newly developed imaging techniques, such as narrow band imaging (NBI, Olympus Optical, Tokyo, Japan), autofluorescence imaging (AFI, Olympus Optical, Tokyo, Japan), flexible spectral imaging colour enhancement (FICE, Fujifilm, Tokyo, Japan), iScan (Pentax Medical, Tokyo, Japan), and confocal laser endomicroscopy (CLE), are now increasingly used in tertiary centres by expert endoscopists for detection of early neoplastic lesions. ${ }^{14} 15$ All these modalities may improve detection of $\mathrm{VL}$ and can also accurately estimate its extension, which is essential for endoscopic treatment, and they are therefore associated with an increased potential for curative treatment.

Endoscopic therapies are the first choice option for curative treatment of early Barrett's neoplasia limited to the mucosal layer, HGD and intramucosal adenocarcinoma (T1m1-T1m3). The aim of endoscopic therapy must be to eradicate the whole Barrett's segment because of the risk of developing synchronous and metachronous lesions in the residual epithelium. ${ }^{16}$

Endoscopic mucosal resection (EMR) of any macroscopic focal lesion followed by eradication of all residual Barrett's segment has become the treatment of choice for HGD and early Barrett's intramucosal cancer. Radiofrequency ablation (RFA) has shown the best efficacy and safety profile in eradicating dysplastic and non-dysplastic Barrett's oesophagus. ${ }^{10} 1718$

Between 50\% and $80 \%$ of Barrett's patients with HGD and no VLs referred for endoscopic treatment to a tertiary referral hospital will have at least one VL when reviewed by expert endoscopists. ${ }^{10} 19$

The presence of superficial lesions within the $\mathrm{BO}$ with HGD carries a higher risk of SM invasion. ${ }^{19}$ Depth of invasion correlates with tumour differentiation grade and lymphovascular involvement, and both of these are well-known risk factors for lymph node metastases. Endoscopic appearance of VLs has predictive value for invasion into the submucosa; ${ }^{20} 21$ Paris 0-IIa and 0-IIb lesions are less likely to contain sub-mucosal invasive cancer. Up to $25 \%$ of $0-$ Is and $0-$ IIc lesions may harbour SM invasion. ${ }^{7}$

To ensure cancers are not inadvertently ablated, a baseline HRE should be performed in all Barrett's patients with HGD before ablative therapy to detect any VL suitable for endoscopic resection. ${ }^{10} 22$

The incidence of metachronous VL in patients referred for treatment with RFA for dysplastic BO and its impact in the treatment outcomes has not been well established.

\section{AIMS}

To evaluate the incidence of metachronous VL's in patients referred for RFA therapy for early Barrett's neoplasia.

\section{PATIENTS AND METHODS}

All patients with dysplastic $\mathrm{BO}$ referred for endoscopic treatment with RFA to our institution were prospectively collected and included for analysis (June 2012 to December 2014). This study was conducted as part of the service evaluation audit; hence, formal ethics application was not required.

Before any ablation treatment, a careful endoscopic examination with white light HRE and AFI followed by NBI was sequentially performed (GIF-FQ260Z; Olympus Optical, Tokyo, Japan) in all patients by an expert endoscopist (KR, JDC, AC). Endoscopic characteristics of the Barrett's segment according to the Prague classification ${ }^{23}$ and endoscopic appearances of all VLs according to the Paris classification ${ }^{7}$ were recorded per procedure and patient.

EMR was performed for all identified VL using the multiband technique (Duette; Cook Endoscopy, Limerick, Ireland) or the cap-assisted technique (Olympus, Tokyo, Japan). Three to six months after the initial EMR, all patients were treated with RFA either with $\mathrm{HALO}^{360}$, $\mathrm{HALO}^{90}, \mathrm{HALO}^{60}$, HALO Ultra or HALO TTS systems (Covidien, Dublin, Ireland). Ablation with argon plasma coagulation (APC) (ERBE Elektromedizin $\mathrm{GmbH}$, Tübingen, Germany) was also used for residual ultrashort Barrett's segments or small islands after RFA and was also considered as an ablation procedure. Repeat ablation sessions were performed at 3-month intervals.

Metachronous lesions during RFA were defined as any VL within the Barrett's segment identified at the first visit for RFA treatment or at any scheduled visit for ablation during the RFA treatment protocol. Endoscopic characteristics according to the Paris classification were recorded. EMR was performed using the same technique described above for all metachronous lesions and final histology was included for analysis.

All endoscopy reports, final histology and staging by $\mathrm{EMR} /$ surgery were evaluated and included for analysis. 


\section{RESULTS}

A total of 50 patients with dysplastic BO referred for RFA and enrolled in the ablation treatment protocol were analysed; median age was 73 years, range 46-83, and $84 \%$ were men. The median length of $\mathrm{BO}$ was $6 \mathrm{~cm}$ (range 1-16 cm).

Initial histology from previous mapping and/or target biopsies was LGD in two patients, HGD in 34, IMC in 13 and SM cancer in one patient. The only patient with SM cancer was referred for surgery and underwent RFA treatment for residual BO after oesophagectomy. Baseline and demographic characteristics are summarised in table 1.

Thirty-eight of 50 patients $(76 \%)$ had a previous EMR due to the presence of VLs before referred for ablation. Twelve patients had a flat Barrett's segment with no previous treatment.

A total of 151 ablation procedures were performed, median per patient 2.68 , range 1-6 sessions. RFA was successfully applied in 139 sessions $\left(\mathrm{HALO}^{360}=29\right.$, $\mathrm{HALO}^{90}=74, \mathrm{HALO}^{60}=9$, HALO Ultra $=15$ and HALO TTS =12). APC was used as a rescue ablation method in 12 patients at the end of the treatment protocol. All of them had a single session with APC and it was their last interventional endoscopy.

Twenty of the 50 patients (40\%) who entered the ablation protocol finished the treatment and are on endoscopic surveillance; no evidence of residual or recurrent dysplasia was noted on biopsies follow-up.

A total of 20 metachronous lesions were identified in 14 patients at the first RFA visit or during the ablation treatment; incidence was $28 \%$. Two patients had two different VLs, one patient had three VLs and two

Table 1 Demographics and Barrett's segment characteristics

\begin{tabular}{ll}
\hline $\mathrm{N}$ & 50 \\
Mean age (years) & 73.5 (range 46-83) \\
Gender (M/F) & $42 / 8$ \\
Barrett's type & \\
$\quad$ Short segment & $4(8 \%)$ \\
$\quad$ Long segment & $46(92 \%)$ \\
Median Barrett's length (cm) & 6 (range 1-16) \\
Visible lesions before RFA & $12(24 \%)$ \\
$\quad$ Yes & $38(76 \%)$ \\
No & \\
Initial histological diagnosis & 2 \\
LGD & 34 \\
HGD & 13 \\
IMC & \\
Treatment & 11 \\
RFA & 38 \\
EMR+RFA & 1 \\
Surgery+RFA
\end{tabular}

EMR, endoscopic mucosal resection; HGD, high-grade dysplasia; IMC, intramucosal cancer; LGD, low-grade dysplasia; RFA, radiofrequency ablation. patients had a VL in two different ablation visits. Endoscopic characteristics according to the Paris classification were $0-\mathrm{Is}=1,0-\mathrm{IIa}=18$ and $0-\mathrm{IIb}=1$.

All metachronous lesions were successfully resected by EMR; final histology per lesion was non-dysplastic $\mathrm{BO}$ in 1, LGD in 1, HGD in 11, IMC in 5 and SM cancer in 2 lesions. Both patients with SM invasion on the rescue EMR specimen had T1sm2 tumours. One was not candidate for surgery and re-entered the RFA treatment due to residual dysplastic BO during the follow-up; the other patient was referred for surgery.

Upstaging after rescue EMR compared with the initial histology was observed in four patients (28\%), histological assessment of EMR specimens showed $\mathrm{SM}$ invasion in 2.

Time of diagnosis of metachronous lesions is shown in table 2; median ablation visit 1.5, mean 2.5. Combined EMR and RFA on the same session were successfully performed in five patients with metachronous lesions (figures 1 and 2).

\section{DISCUSSION}

Combined endoscopic treatment with EMR of any macroscopic lesion followed by complete eradication of the residual Barrett's segment with RFA has become the treatment of choice for early Barrett's neoplasia. The aim of the endoscopic therapy is the eradication of the whole Barrett's segment due to the high risk of synchronous and metachronous lesions. Many clinical trials from Europe and the USA, ${ }^{18} 22$ 24-26 data from the UK Halo RFA Registry, ${ }^{10}$ and a recently published meta-analysis ${ }^{17}$ have shown excellent rates of complete eradication of dysplasia and IM with very low recurrence rates up to 5 years of follow-up.

There are no published studies clearly focused on metachronous or recurrent macroscopic lesions during the ablation treatment. Several studies conducted by the Amsterdam group showed incidence rates of metachronous lesions during treatment or follow-up period between 4\% and 9\%. ${ }^{18} 222425$ Most recently, data from the UK Halo RFA Registry, including a total of 335 patients, reported an incidence of metachronous lesions during RFA treatment protocol of $10 \% .^{10}$

Our study has showed that up to $28 \%$ of patients enrolled in an RFA programme were diagnosed to

Table 2 Time of diagnosis of metachronous lesions $(n=16)$

\begin{tabular}{ll}
\hline Ablation visit 1 & $6(37.5 \%)$ \\
Ablation visit 2 & $3(18.7 \%)$ \\
Ablation visit 3 & $4(25 \%)$ \\
Ablation visit 4 & $3(18.7 \%)$ \\
Ablation visit 5 & 0 \\
Ablation visit 6 & 0 \\
\hline
\end{tabular}




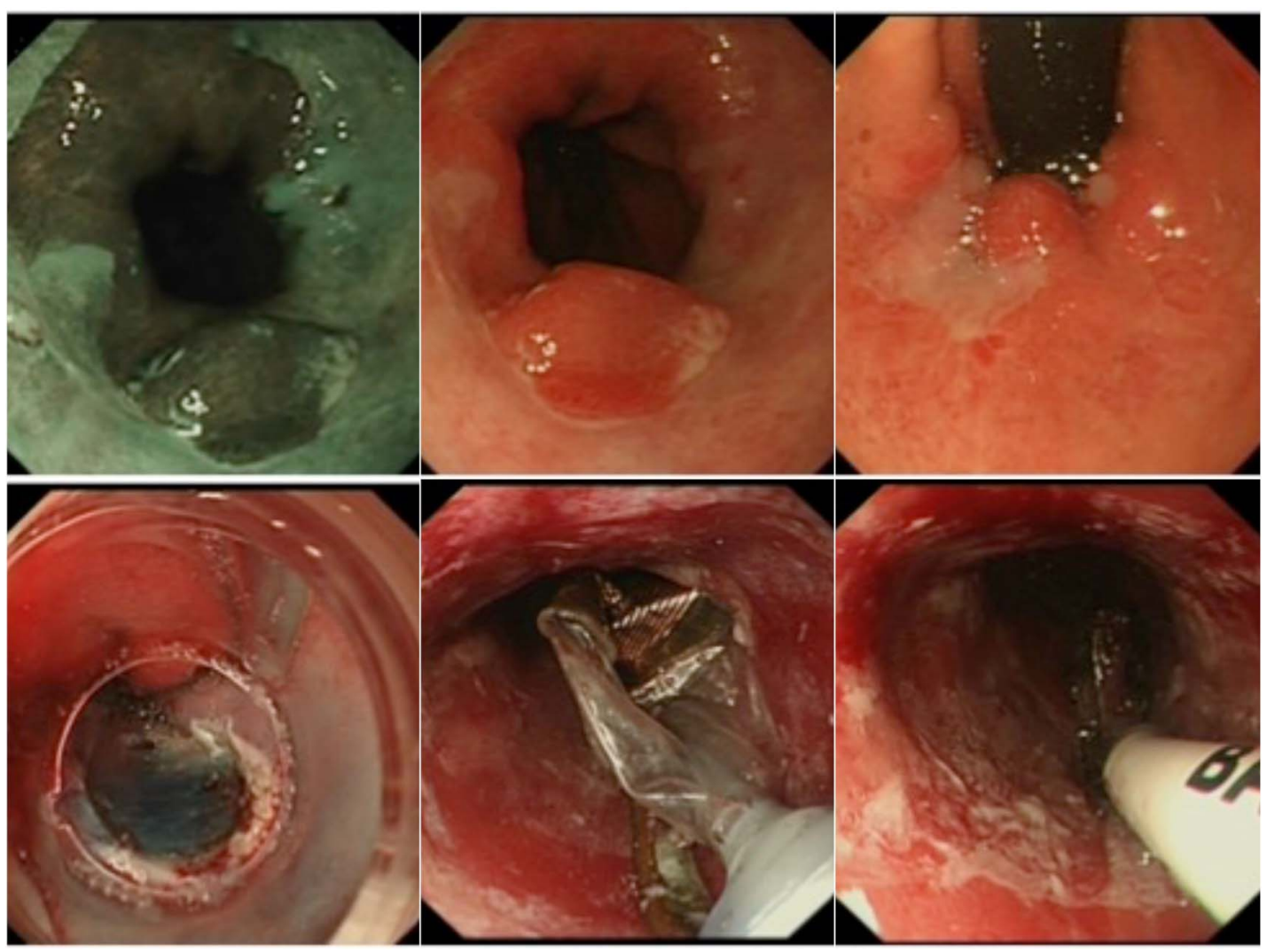

Figure 1 0-Ila metachronous lesion resected by endoscopic mucosal resection (IMC/T1a). Radiofrequency ablation HALO360 performed on the same session.

have metachronous lesions within the Barrett's segment at their first visit for RFA or during the treatment period. The diagnosis of new VL during RFA treatment had a big impact in the therapeutic management of two patients who had SM invasion in the rescue EMR specimens.

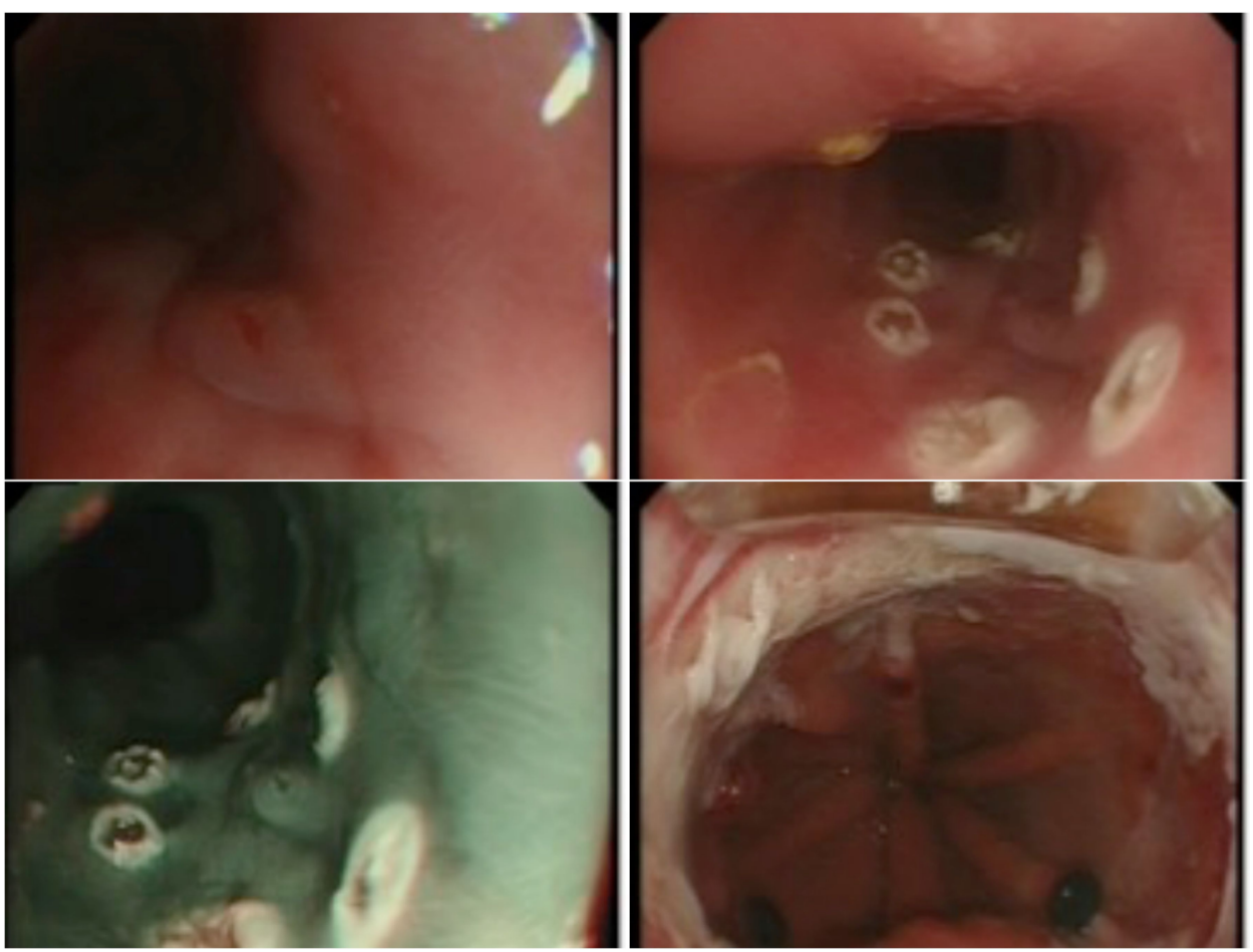

Figure 2 0-llb metachronous lesion resected by endoscopic mucosal resection (IMC/T1a). Radiofrequency ablation HALO90 performed on the same session. 
Main limitation of our study is that this is a singlecentre experience and shows data from a small case series.

This high-incidence rate highlights the importance of a meticulous examination to identify and resect any superficial lesion before every ablation session to avoid ablation of deep invasive cancer.

Treatment with RFA for early Barrett's neoplasia should be performed in tertiary referral centres by expert endoscopists with an adequate training in advanced endoscopic imaging and EMR facilities and expertise.

\section{Key messages}

What is already known on this topic

- EMR of any macroscopic focal lesion followed by eradication of all residual Barrett's segment with RFA has become the treatment of choice for HGD and mucosal cancer in Barrett's oesophagus.

\section{What this study adds}

- We found a high incidence of metachronous visible lesions in patients referred for RFA or in patients undergoing ablation therapy.

\section{How might it impact on clinical practice in the fore- seeable future}

- Meticulous examination to identify and resect any superficial lesion before every ablation session is mandatory. RFA for early Barrett's neoplasia should be performed in tertiary referral centres by expert endoscopists with an adequate training in advanced endoscopic imaging and EMR facilities and expertise.

Contributors JO-F-S and KR: Involved in substantial contributions to manuscript concept and design. JO-F-S: Performed data collection, analysis and interpretation. All authors participated in drafting the article, revising and approval of content for final version.

Competing interests KR has received research support, educational grants, speaker honoraria, and consultancy fees from Olympus Keymed, Pentax, Cook Medical, Covidien and Boston Scientific.

Provenance and peer review Not commissioned; externally peer reviewed.

Open Access This is an Open Access article distributed in accordance with the Creative Commons Attribution Non Commercial (CC BY-NC 4.0) license, which permits others to distribute, remix, adapt, build upon this work noncommercially, and license their derivative works on different terms, provided the original work is properly cited and the use is non-commercial. See: http://creativecommons.org/licenses/bync/4.0/

\section{REFERENCES}

1 Sharma P. Clinical practice: Barrett's esophagus. N Engl J Med 2009;361:2548-56.

2 Buttar NS, Wang KK, Sebo TJ, et al. Extent of high-grade dysplasia in Barrett's esophagus correlates with risk of adenocarcinoma. Gastroenterology 2001;120:1630-9.
3 Weston AP, Sharma P, Topalovski M, et al. Long-term follow-up of Barrett's high-grade dysplasia. Am J Gastroenterol 2000;95:1888-93.

4 Stolte M, Kirtil T, Oellig F, et al. The pattern of invasion of early carcinomas in Barrett's esophagus is dependent on the depth of infiltration. Pathol Res Pract 2010;206:300-4.

5 Feith M, Stein HJ, Siewert JR. Pattern of lymphatic spread of Barrett's cancer. World J Surg 2003;27:1052-7.

6 Bergman JJ. Endoscopic treatment of high-grade intraepithelial neoplasia and early cancer in Barrett oesophagus. Best Pract Res Clin Gastroenterol 2005;19:889-907.

7 Endoscopic Classification Review Group. Update on the Paris classification of superficial neoplastic lesions in the digestive tract. Endoscopy 2005;37:570-8.

8 Levine DS, Haggitt RC, Blount PL, et al. An endoscopic biopsy protocol can differentiate high-grade dysplasia from early adenocarcinoma in Barrett's esophagus. Gastroenterology 1993;105:40-50.

9 Fitzgerald RC, di Pietro M, Ragunath K, et al. British Society of Gastroenterology guidelines on the diagnosis and management of Barrett's oesophagus. Gut 2014;63:7-42.

10 Haidry RJ, Dunn JM, Butt MA, et al. Radiofrequency ablation and endoscopic mucosal resection for dysplastic Barrett's esophagus and early esophageal adenocarcinoma: outcomes of the UK National Halo RFA Registry. Gastroenterology 2013;145:87-95.

11 Pouw RE, Heldoorn N, Herrero LA, et al. Do we still need EUS in the workup of patients with early esophageal neoplasia? A retrospective analysis of 131 cases. Gastrointest Endosc 2011;73:662-8.

12 Thomas T, Gilbert D, Kaye PV, et al. High-resolution endoscopy and endoscopic ultrasound for evaluation of early neoplasia in Barrett's esophagus. Surg Endosc 2010;24:1110-16.

13 Ortiz-Fernández-Sordo J, Konda VJ, Chennat J, et al. Is Endoscopic Ultrasound (EUS) necessary in the pre-therapeutic assessment of Barrett's esophagus with early neoplasia? J Gastrointest Oncol 2012;3:314-21.

14 Curvers WL, Kiesslich R, Bergman JJ. Novel imaging modalities in the detection of oesophageal neoplasia. Best Pract Res Clin Gastroenterol 2008;22:687-720.

15 Subramanian V, Ragunath K. Advanced endoscopic imaging: a review of commercially available technologies. Clin Gastr Hepatol 2014;12:368-76.

16 Hage M, Siersema PD, Vissers KJ, et al. Molecular evaluation of ablative therapy of Barrett's oesophagus. J Pathol 2005;205:57-64.

17 Orman ES, Li N, Shaheen NJ. Efficacy and durability of radiofrequency ablation for Barrett's esophagus: systematic review and meta-analysis. Clin Gastroenterol Hepatol 2013;11:1245-55

18 Phoa KN, Pouw RE, van Vilsteren FG, et al. Remission of Barrett's esophagus with early neoplasia 5 years after radiofrequency ablation with endoscopic resection: a Netherlands cohort study. Gastroenterology 2013; 145:96-104.

19 Pech O, Gossner L, Manner H, et al. Prospective evaluation of the macroscopic types and location of early Barrett's neoplasia in 380 lesions. Endoscopy 2007;39:588-93.

20 Zemler B, May A, Ell C, et al. Early Barrett's carcinoma: the depth of infiltration of the tumour correlates with the degree of differentiation, the incidence of lymphatic vessel and venous invasion. Virchows Arch 2010;456:609-14. 
21 Alvarez Herrero L, Pouw RE, van Vilsteren FG, et al. Risk of lymph node metastasis associated with deeper invasion by early adenocarcinoma of the esophagus and cardia: study based on endoscopic resection specimens. Endoscopy 2010;42:1030-6.

22 van Vilsteren FG, Pouw RE, Seewald S, et al. Stepwise radical endoscopic resection versus radiofrequency ablation for Barrett's oesophagus with high-grade dysplasia or early cancer: a multicentre randomised trial. Gut 2011;60:765-73.

23 Sharma P, Dent J, Armstrong D, et al. The development and validation of an endoscopic grading system for Barrett's esophagus: the Prague C \& M criteria. Gastroenterology 2006;131:1392-9.
24 Pouw RE, Gondrie JJ, Sondermeijer CM, et al. Eradication of Barrett esophagus with early neoplasia by radiofrequency ablation, with or without endoscopic resection. J Gastrointest Surg 2008;12:1627-37.

25 Pouw RE, Wirths K, Eisendrath P, et al. Efficacy of radiofrequency ablation combined with endoscopic resection for Barrett's esophagus with early neoplasia. Clin Gastroenterol Hepatol 2010;8:23-9.

26 Orman ES, Kim HP, Bulsiewicz WJ, et al. Intestinal metaplasia recurs infrequently in patients successfully treated for Barrett's esophagus with radiofrequency ablation. Am J Gastroenterol 2013;108:187-95. 